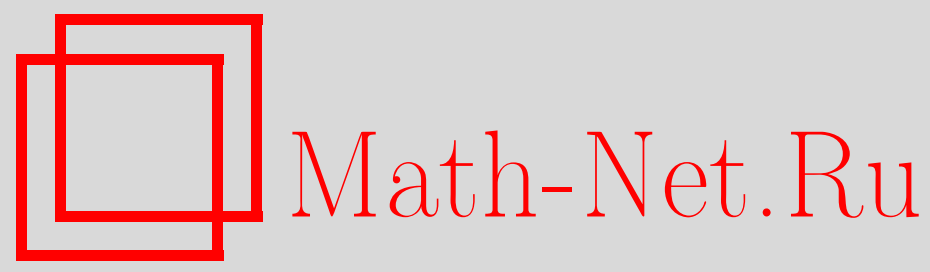

И. А. Бадеха, П. В. Ролдугин, О плотности графов, в которых каждое ребро входит хотя бы в две максимальные клики, Дискрет. матем., 2013, том 25, выпуск 3, 7-21

DOI: https://doi.org/10.4213/dm1244

Использование Общероссийского математического портала Math-Net.Ru подразумевает, что вы прочитали и согласны с пользовательским соглашением http://www.mathnet.ru/rus/agreement

Параметры загрузки:

IP: 3.95 .254 .165

26 апреля 2023 г., $11: 58: 17$

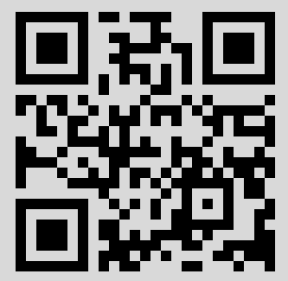




\title{
О плотности графов, в которых каждое ребро входит хотя бы в две максимальные клики
}

\author{
( 2013 г. И. А. Бадеха, П. В. Ролдугин
}

\begin{abstract}
В статье найдены возможные значения плотности графов, в которых каждое ребро лежит не менее, чем в двух максимальных кликах. Описаны графы, на которых достигаются максимальные значения плотности.
\end{abstract}

\section{Введение}

Кликой в графе $G$ называется любой полный подграф графа $G$; полные подграфы, максимальные по включению, называются максимальными кликами графа $G$. Порядок клики - это число её вершин. Клики порядка 3 будем называть треугольниками. Плотностью $\rho(G)$ графа $G$ называется порядок наибольшей клики графа $G$; наибольшую среди степеней вершин графа $G$ обозначим $\Delta(G)$ и назовём максимальной степенью графа. Когда это очевидно из контекста, говоря о максимальной клике, слово «максимальная» будем опускать; кроме того, множество вершин клики $K$ будем обозначать также буквой $K$, подразумевая, что любые две вершины из $K$ смежны. Для вершины $x$ через $N(x)$ обозначим ее окружение, то есть множество вершин графа, смежных с вершиной $x$. Используем $N^{*}(x)$ для обозначения замкнутого окружения, то есть $N^{*}(x)=N(x) \bigcup\{x\}$. В случаях, когда необходимо уточнить граф, в котором рассматривается окружение (замкнутое окружение), используем обозначение $N_{G}(x)\left(N_{G}^{*}(x)\right)$. Подграфом графа $G$, порожденным множеством вершин $M$ (или просто порожденным подграфом), назовем граф, получаемый из исходного графа $G$ удалением вершин, не лежащих в $M$, и инцидентных им ребер.

Ребро, лежащее только в одной максимальной клике графа $G$, назовем собственным ребром графа $G$. Изучение графов, не содержащих собственных ребер, в частности, вызвано ролью таких графов в задаче поиска покрытия всех ребер графа наименьшим числом максимальных клик (см., например, [1-5]). В [1] показано, что клика входит в любое покрытие графа тогда и только тогда, когда клика содержит собственное ребро. То есть в определенном смысле собственные ребра графа позволяют «упростить» задачу поиска покрытия, указывая клики, которые обязательно будут в искомом покрытии.

Понятно, что граф, не содержащий собственных ребер, не может иметь очень большое значение плотности: например, в полном графе каждое ребро является 
собственным. Основной целью настоящей работы является изучение спектра возможных значений плотности графов без собственных ребер. Однако без дополнительных ограничений на граф эта задача тривиальна, ввиду следующего свойства: к графу без собственных ребер можно добавить «копию» любой вершины, и полученный граф также не будет иметь собственных ребер. Это свойство отмечено, например, в работе [3]. Сформулируем его более строго и, поскольку обоснование этого свойства несложно, для полноты изложения приведем доказательство.

Утверждение 1. Пусть $G$ - граф порядка р. Построим граф $G^{\prime}$, выбрав в графе $G$ произвольную неизолированную вершину $x$, добавив новую вершину $x^{\prime}$ и соединив ребрами вершину $x^{\prime}$ со всеми вершинами замкнутого окружения $N^{*}(x)$ вершинь $x$. Тогда:

1) множество всех максимальных клик графа $G^{\prime}$ получается из множества всех максимальных клик графа $G$ добавлением вершины $x^{\prime}$ в те клики, которые содержат вериину $x$;

2) граф $G^{\prime}$ является графом без собственных ребер тогда и только тогда, когда графб $G$ не содержит собственных ребер.

Доказательство. По построению любая вершина $y$, отличная от $x$ и $x^{\prime}$, смежна с вершиной $x$ тогда и только тогда, когда $y$ смежна с $x^{\prime}$. Так как вершины $x$ и $x^{\prime}$ смежны в графе $G^{\prime}$, то вершина $x$ лежит в произвольной максимальной клике $L$ графа $G^{\prime}$ тогда и только тогда, когда $x^{\prime}$ лежит в клике $l$. Из сказанного следует 1.

Из 1 вытекает, что любое ребро графа $G$ лежит только в одной максимальной клике графа $G$ тогда и только тогда, когда это ребро лежит в одной максимальной клике графа $G^{\prime}$. Аналогично, ребро вида $\left(x^{\prime}, a\right)$, где $a$ - вершина графа $G$, отличная от $x$, лежит в одной клике графа $G^{\prime}$ тогда и только тогда, когда ребро $(x, a)$ лежит в одной клике графа $G$. Наконец, поскольку по условию в графе $G$ вершина $x$ не является изолированной, то существует вершина $z$, смежная с $x$, и тогда ребро $\left(x, x^{\prime}\right)$ является собственным в графе $G^{\prime}$ тогда и только тогда, когда ребро $(x, z)$ является собственным в графе $G$.

Из утверждения 1 можно сделать следующий простой вывод: если существует граф без собственных ребер плотности $\rho>1$, то существуют графы без собственных ребер плотности $\rho+1, \rho+2$ и так далее, поскольку всегда можно в наибольшую клику графа добавлять "копии" любых ее вершин.

Для получения более содержательных результатов далее в статье будем рассматривать графы, не имеющие "копий" вершин. Сформулируем это требование строго. Введем на множестве вершин графа $G$ отношение эквивалентности. Две вершины $x$ и $y$ называются эквивалентнъми, если $N^{*}(x)=N^{*}(y)$. Очевидно, что эквивалентные вершины $x$ и $y$ обязаны быть смежными. Далее графы, в которых каждый класс эквивалентности содержит ровно одну вершину, назовем сжатым графом.

Очевидно также, что свойства сжатости или отсутствия у графа свободных ребер имеют место тогда и только тогда, когда этими свойствами обладает каждая компонента связности графа. Поэтому целесообразно ограничиться изучением только связных графов.

Обозначим класс всех связных сжатых графов без собственных ребер через э. Для упрощения формулировок считаем по определению, что граф, состоящий из одной вершины, не лежит в классе $\Im$.

Приведем в качестве примера граф $B$ порядка 6 , изображенный на рис. 1 . Этот граф получается при удалении из полного графа на 6 вершинах любых трех попар- 
но не смежных ребер. Граф $B$ сжат и свободен от собственных ребер, кроме того, $\Delta(B)=4$ и $\rho(B)=3$.

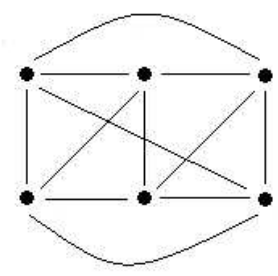

Рис. 1. Граф $B$

Поскольку в произвольном графе вершина любой клики смежна со всеми остальными вершинами этой клики, верно неравенство $\rho(G) \leqslant \Delta(G)+1$. Ниже доказано (см. утверждение 5$)$, что для графов из класса $\Im$ выполнено более сильное неравенство $\rho(G) \leqslant \Delta(G)-1$.

Основными результатами данной работы являются следующие утверждения. Напомним, что доминирующей вершиной графа называется вершина, смежная со всеми остальными вершинами графа.

Теорема 1. Граф из класса $\Im$ с точностъю до изоморфизма совпадает с графом $B$, если он удовлетворяет хотя бы одному из условий:

1) плотность графа на единииу меньше максимальной степени графа;

2) максимальная степень графа не превышает 4;

3) порядок графа не превышает 6.

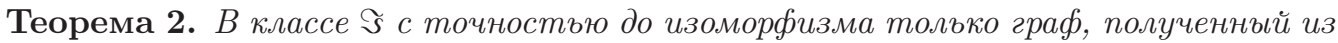
графа В добавлением доминирующей вериинь, удовлетворяет одновременно двум условиям: плотность графа на два меньше максимальной степени графа и существует единственная вершина максимальной степени.

Теорема 3. Для любых натуральных $\rho$ и $\Delta$, удовлетворяющих условиям $\rho \geqslant 3$, $\Delta(G) \geqslant 2 \rho-2$, в классе $\Im$ существует граф плотности $\rho$ и максимальной степени $\Delta$.

\section{Экстремальное значение плотности}

При доказательствах будем часто использовать следующее характеристическое свойство собственного ребра, доказанное в [1].

Утверждение 2. Ребро е графа $G$ является собственным тогда и только тогда, когда множество вершин графа $G$, смежных одновременно с обоими концами ребра е, порождает полный подграф в $G$.

Далее изложим свойства графов из класса $\Im$ в ряде последовательных утверждений.

Утверждение 3. В графе $G \in \Im$ для любых двух различных вершин произвольной максимальной клики $K$ существует смежная с ними обоими вершина, не лежащая в клике $K$. 
Доказательство. Пусть $x$ и $y$-различные вершины максимальной клики $K$ графа $G$. Ребро $(x, y)$ не является собственным ребром клики $K$, то есть существует другая максимальная клика $L$, содержащая вершины $x$ и $y$. Так как клика $L$ не совпадает с $K$, то в силу их максимальности найдется вершина $z$, лежащая в $L$ и не лежащая в $K$. Вершина $z$ и есть искомая смежная с $x$ и $y$ вершина, не лежащая в клике $K$.

Утверждение 4. В графе $G \in \Im$ окружение каждой вериины максимальной клики $K$ содержит две смежные вериины, не лежащие в клике $K$.

Доказательство. Предположим, что в максимальной клике $K$ существует вершина $x$, окружением которой является $K \backslash\{x\}$. Тогда вершина $x$ не лежит ни в какой другой максимальной клике, кроме клики $K$. По условию граф связен и не состоит из одной изолированной вершины, поэтому число вершин в $K$ больше 1 . Значит, существует хотя бы одно ребро $(x, y)$, где $y \in K$. Поскольку вершина $x$ лежит только в клике $K$, то и ребро $(x, y)$ принадлежит только клике $K$ и поэтому является ее собственным ребром, что противоречит условию.

Предположим теперь, что $N^{*}(x)=K \bigcup\{b\}$, где вершина $b$ не лежит в клике $K$. Рассмотрим произвольную вершину $y$ клики $K$, отличную от $x$. По утверждению 3 вершина $y$ смежна с $b$, так как $b$ - единственная вершина вне клики $K$, смежная с $x$. Значит, все вершины клики $K$ смежны с вершиной $b$, что противоречит условию максимальности клики $K$.

Пусть теперь $N^{*}(x)=K \bigcup B, K \bigcap B=\varnothing$ и $|B| \geqslant 2$, то есть окружение вершины $x$ содержит не менее двух вершин, не лежащих в клике $K$; пусть $y \in B$ - одна из таких вершин. Если $y$ не смежна ни с какими другими вершинами из $b$, то все вершины, смежные одновременно с $x$ и $y$, лежат в клике $K$. То есть все такие вершины смежны и по утверждению 2 ребро $(x, y)$ является собственным ребром, что противоречит условию.

Утверждение 5. Для графа $G \in \Im$ верны неравенства $3 \leqslant \rho(G) \leqslant \Delta(G)-1$.

Доказательство. Пусть $K$ - клика размера $\rho=\rho(G)$ в графе $G$. Возьмем произвольную вершину $x$ из клики $K$. Вершина $x$, очевидно, смежна со всеми вершинами клики $K$ (то есть с $\rho-1$ вершинами) и по утверждению 4 еще по крайней мере с двумя вершинами, не лежащими в клике $K$. Имеем $\operatorname{deg} x \geqslant \rho-1+2=\rho(G)+1$. Так как $\Delta(G) \geqslant \operatorname{deg} x$, то $\Delta(G) \geqslant \rho(G)+1$.

Для графа $G \in \Im$ выполнено неравенство $\rho(G) \geqslant 3$, поскольку в противном случае граф не содержит треугольников, а следовательно, каждое ребро является собственным ребром максимальной клики, состоящей из одного ребра, что противоречит условию.

Утверждение 6. Если в графе $G \in \Im$ все вершины некоторой максимальной клики размера q имеют порядок не более $q+1$, mо $q=3$ и граф $G$ содержит порожденный подграф, изоморфный графу $B$ (рис. 1).

Доказательство. В графе $G$ нет максимальных клик порядка 1, поскольку в противном случае в силу связности весь граф состоял бы из одной вершины, а по определению такой граф не считается сжатым графом без собственных ребер. Рассмотрим в графе $G$ максимальную клику $K$ размера $q$, все вершины которой имеют порядок не более $q+1$. Согласно сказанному $q>1$. 
По утверждению 4 произвольная вершина клики $K$ смежна как минимум с двумя вершинами, не лежащими в клике $K$. Поскольку вершина смежна также с $q-1$ другими вершинами клики $K, \operatorname{deg} a \geqslant q-1+2=q+1$. Так как $\operatorname{deg} a \leqslant$ $q+1$, то $\operatorname{deg} a=q+1$; это означает, что любая вершина клики $K$ смежна с ровно с двумя вершинами, не лежащими в клике $K$. Кроме того, по утверждению 4 эти две вершины смежные.

Пусть $a_{1}-$ произвольная вершина клики $K$. Обозначим далее две смежные вершины, не лежащие в клике $K$ и смежные с $a_{1}$, через $b_{1}$ и $b_{2}$ (рис. $2 \mathrm{a}$ ). Так как $q>1$, то найдется $a_{2}$ - вершина клики $K$, отличная от $a_{1}$. По утверждению 3 у вершин $a_{1}$ и $a_{2}$ есть смежная с ними вершина, не лежащая в клике $K$. Без ограничения общности считаем, что это вершина $b_{2}$.

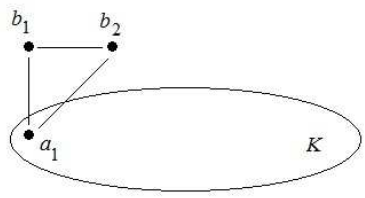

a)

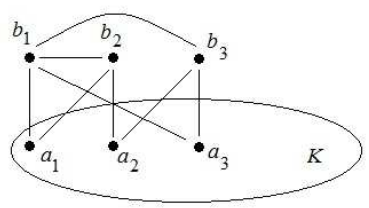

г)

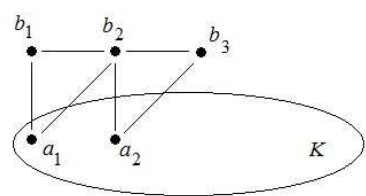

б)

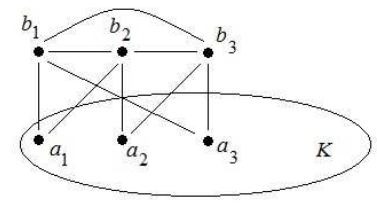

д)

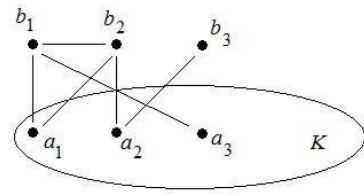

B)

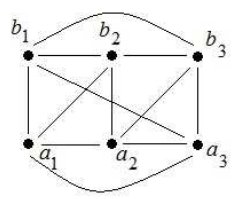

e)

Рис. 2.

По доказанному выше вершина $a_{2}$ смежна ровно с двумя вершинами, не лежащими в клике $K$ (одна из этих вершин - $b_{2}$ ). Если вершина $a_{2}$ смежна и с $b_{1}$, то $N^{*}\left(a_{2}\right)=K \bigcup\left\{b_{1}, b_{2}\right\}=N^{*}\left(a_{1}\right)$; это означает, что вершины $a_{1}$ и $a_{2}$ эквивалентны, что противоречит сжатости графа $G$. Следовательно, вершина $a_{2}$ соединена ребрами с двумя смежными вершинами, не лежащими в клике $K$ : это вершина $b_{2}$ и вершина $b_{3}$, отличная от $b_{1}$ (рис.2б).

Предположим, что $q=2$, то есть что клика $K$ содержит только вершины $a_{1}$ и $a_{2}$. Но тогда клика $K$ целиком содержится в треугольнике $a_{1}, a_{2}, b_{2}$. Следовательно, $q>2$.

Рассмотрим вершины клики $K$, отличные от вершин $a_{1}$ и $a_{2}$. Среди них найдется вершина, смежная с $b_{1}$; в противном случае ребро $\left(a_{1}, b_{1}\right)$ лежит в единственной максимальной клике, образованной вершинами $a_{1}, b_{1}, b_{2}$, то есть является собственным ребром. Обозначим такую вершину через $a_{3}$ (рис. 2в). По утверждению 3 у вершин $a_{2}$ и $a_{3}$ есть смежная с ними вершина, не лежащая в клике $K$. Это не может быть вершина $b_{2}$, поскольку в этом случае $N^{*}\left(a_{3}\right)=K \bigcup\left\{b_{1}, b_{2}\right\}=N^{*}\left(a_{1}\right)$, то есть вершины $a_{1}$ и $a_{2}$ эквивалентны, что невозможно в силу сжатости графа $G$. Следовательно, это вершина $b_{3}$. Поскольку из вершин вне клики $K$ с вершиной $a_{3}$ смежны только $b_{1}$ и $b_{3}$, по утверждению 4 вершины $b_{1}$ и $b_{3}$ смежны (рис. 2г).

Если в графе отсутствует ребро $\left(b_{2}, b_{3}\right)$, то все вершины, одновременно смежные с $a_{2}$ и с $b_{2}$, лежат в клике $K$, то есть все смежны. Тогда по утверждению 2 ребро 
$\left(a_{2}, b_{2}\right)$ является собственным. Получаем противоречие и, следовательно, вершины $b_{2}$ и $b_{3}$ смежны (рис. 2д).

Пусть в клике $K$ существует вершина $a_{4}$, отличная от вершин $a_{1}, a_{2}, a_{3}$. Из рассуждений выше следует, что вне клики $K$ найдется ровно две вершины $z_{1}, z_{2}$, смежные с $a_{4}$. По утверждению 3 в эти две вершины должны входить $b_{1}$ или $b_{2}$ (должна существовать вершина вне клики $K$, смежная с $a_{4}$ и с $a_{1}$ ), $b_{2}$ или $b_{3}$ (аналогично для вершины $\left.a_{2}\right), b_{1}$ или $b_{3}$ (то же для $a_{3}$ ). То есть возможен один из трех вариантов: $\left\{z_{1}, z_{2}\right\}=\left\{b_{1}, b_{2}\right\},\left\{z_{1}, z_{2}\right\}=\left\{b_{2}, b_{3}\right\},\left\{z_{1}, z_{2}\right\}=\left\{b_{1}, b_{3}\right\}$. В любом из трех случаев вершина $a_{4}$ оказывается эквивалентной одной из вершин $a_{1}, a_{2}, a_{3}$, что противоречит сжатости графа $G$. Следовательно, в клике $K$ ровно 3 вершины, то есть $q=3$ (рис. 2е).

Степени вершин $a_{1}, a_{2}, a_{3}$ равны $q+1=4$. По этой причине в графе $G$ нет ребер $\left(a_{1}, b_{3}\right),\left(a_{2}, b_{1}\right),\left(a_{3}, b_{2}\right)$. Удалив из графа $G$ все вершины, кроме $a_{1}, a_{2}, a_{3}, b_{1}$, $b_{2}, b_{3}$, и инцидентные удаляемым вершинам ребра, получим порожденный подграф, приведенный на рис. 2е. Нетрудно убедиться, что этот граф изоморфен графу $B$.

Утверждение 7. Если для графа $G \in \Im$ выполнено равенство $\rho(G)=\Delta(G)-1$, то $\Delta(G)=4$ и граф изоморфен графу $B$ (рис. 1).

Доказательство. Рассмотрим максимальную клику $K$ размера $\rho(G)$ в графе $G$. Все вершины графа (и вершины этой клики в частности) имеют порядок не более $\Delta(G)=\rho(G)+1$. Значит, к графу $G$ и клике $K$ применимо утверждение 6 . Получаем, что $\rho(G)=3$ (поэтому $\Delta(G)=4$ ) и что графе $G$ есть порожденный подграф $B^{\prime}$, изоморфный графу $B$. Все вершины графа $B$ имеют степень 4 . Если в графе $G$ существует вершина, не лежащая в порожденном подграфе $B^{\prime}$, то она, в силу связности графа $G$, должна быть соединена цепью с какой-то вершиной $a$, принадлежащей подграфу $B^{\prime}$; однако тогда степень вершины будет больше 4, что невозможно. Следовательно, граф $G$ совпадает с подграфом $B^{\prime}$ и поэтому изоморфен графу $B$.

Утверждение 8. С точностью до изоморфизма в классе э существует единственный граф с условием $\Delta(G) \leqslant 4$ : это граф $B$.

Доказательство. По утверждению 5 для графа $G \in \Im$ выполнено неравенство $\rho(G) \geqslant 3$. Если $\Delta(G) \leqslant 3$, то, согласно утверждению $5, \rho(G) \leqslant 2$, что приводит к противоречию. Если $\Delta(G)=4$, то $\rho(G) \leqslant 3$ и, следовательно, $\rho(G)=3$. Но тогда $\rho(G)=\Delta(G)-1$ и по утверждению 7 граф $G$ изоморфен графу $B$.

Далее рассмотрим случай, когда $\rho(G)=\Delta(G)-2$.

Утверждение 9. Если $\rho(G)=\Delta(G)-2$ и в графе $G \in \Im$ существует ровно одна вериина максимальной степени, то эта вершина принадлежит наибольшей клике графба $G$.

Доказательство. Обозначим единственную вершину в графе $G$, имеющую степень $\Delta(G)$, через $x$. Через $K$ обозначим максимальную клику графа $G$, имеющую наибольший размер $\rho(G)$.

Предположим сначала, что вершина $x$ не лежит в клике $K$. Тогда все вершины клики $K$ имеют порядок не более $\Delta(G)-1=\rho(G)+1$. Применяя утверждение 6 , получаем, что $\rho(G)=3, \Delta(G)=5$ и в графе $G$ есть порожденный подграф $B^{\prime}$, изоморфный графу $b$. Если вершина $x$ не лежит в подграфе $B^{\prime}$, то из связности графа $G$ получаем, что какая-то вершина $а$ подграфа $B^{\prime}$ должна быть соединена 
ребром с некоторой вершиной $b$, не лежащей в подграфе $B^{\prime}$. Тогда $\operatorname{deg} a \geqslant 5$, a поскольку $\Delta(G)=5$, то $\operatorname{deg} a=5$. Но вершина максимальной степени только одна, значит, вершина и есть вершина $x$. Следовательно, остальные вершины подграфа $B^{\prime}$ имеют степень 4 и поэтому не соединены ребрами с вершинами, не лежащими в этом подграфе. Но ребро $(a, b)$ в этом случае является собственным ребром клики, состоящей из одного этого ребра, поскольку все вершины, смежные с $a$ и отличные от $b$, лежат в подграфе $B^{\prime}$ и поэтому не смежны с вершиной $b$. Получаем противоречие, следовательно, вершина $x$ обязана лежать в клике $K$.

Утверждение 10. Если $\rho(G)=\Delta(G)-2$ и $x$ является единственной вершиной максимальной степени графа $G \in \Im$, то для любой вершины у, лежащей в наибольшей клике графа $G$, верно включение $N^{*}(y) \subset N^{*}(x)$.

Доказательство. Пусть $K$ - наибольшая клика графа $G$. По предыдущему утверждению $x$ лежит в клике $K$. Вершина $x$ имеет порядок $\Delta(G)=\rho(G)+2$, значит, она смежна ровно с тремя вершинами графа $G$, не лежащими в клике $K$. Обозначим эти вершины через $a, b, c$.

Рассмотрим произвольную вершину $v$ клики $K$, отличную от вершины $x$. Согласно утверждению 4 найдется не менее двух вершин, не лежащих в клике $K$ и смежных с $v$. Тогда $\operatorname{deg} v \geqslant \rho(G)+1$, а поскольку существует только одна вершина со степенью $\Delta(G)=\rho(G)+2$, то $\operatorname{deg} v=\rho(G)+1$. То есть существует ровно две вершины вне клики $K$, смежные с $v$, причем по утверждению 4 эти две вершины смежны.

Снова рассмотрим произвольную вершину $y$ клики $K$, отличную от вершины $x$. Обозначим две смежные вершины вне клики $K$, соединенные с $y$, через $a_{1}, a_{2}$. Поскольку $N^{*}(y)=K \bigcup\left\{a_{1}, a_{2}\right\}$ и $N^{*}(y)=K \bigcup\{a, b, c\}$, для доказательства утверждения надо показать, что $\left\{a_{1}, a_{2}\right\} \subset\{a, b, c\}$.

Предположим противное, то есть, что $\left\{a_{1}, a_{2}\right\} \not \subset\{a, b, c\}$. По утверждению 3 есть вершина, не лежащая в клике $K$ и смежная с $x$ и с $y$, то есть $\left\{a_{1}, a_{2}\right\} \bigcap\{a, b, c\} \neq \varnothing$. Без ограничения общности считаем, что в $\left\{a_{1}, a_{2}\right\}$ лежит $a$, то есть $\left\{a_{1}, a_{2}\right\}=\{a, d\}$, где $d \notin\{a, b, c\}$ (см. рис 3a).

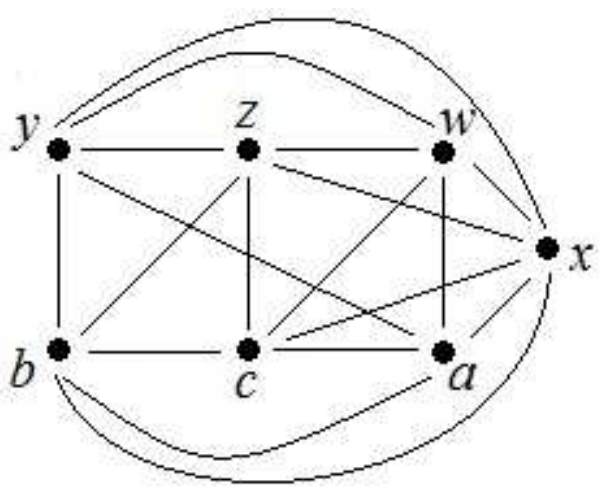

Рис. 3.

Рассмотрим ребро $(y, d)$. Вершины, образующие треугольник с вершинами $\{y, d\}$ - это вершина $a$ и вершины клики $K$, смежные с $d$ (если такие существуют). Если с вершинами $\{y, d\}$ образует треугольник только вершина $a$, то ребро $(y, d)$ является 
собственным; следовательно, в клике $K$ должна существовать вершина $z$, смежная с $d$. Очевидно, что $z$ отлична от $x$, так как вершины $x$ и $d-$ не смежные. Как доказано выше, с вершиной $z$ смежны ровно две вершины, не лежащие в клике $K$ (и эти две вершины соединены ребром). Одна из этих вершин $d$. Другая, по утверждению 3 , должна принадлежать множеству $\{a, b, c\}$. Вершина $a$ не подходит, поскольку тогда вершины $y$ и $z$ оказываются эквивалентны: $N^{*}(y)=K \bigcup\{a, d\}=N^{*}(z)$. Значит, с вершиной $z$ смежна либо $b$, либо $c$; пусть, без ограничения общности, это будет вершина $b$. Как указано выше, вершины $b$ и $d$ смежные (рис. 3б).

Теперь предположим, что в клике $K$ нет вершин, отличных от $x, y, z$. Но тогда $\rho(G)=3$ и $\Delta(G)=\rho(G)+2=5$. Заметим, что с вершиной $d$ уже связаны ребрами 4 вершины, поэтому $\operatorname{deg} d \geqslant 4$, а поскольку $x$ единственная вершина, удовлетворяющая условию $\operatorname{deg} x>4$, то $\operatorname{deg} d=4$. Значит, указанные на рис. 3б ребра, начинающиеся в вершине $d$, - это все ребра, инцидентные вершине $d$ в графе $G$. Но тогда в графе $G$ только одна вершина $y$ смежна с обоими концами ребра $(a, d)$. По утверждению 2 ребро $(a, d)$ собственное, что противоречит условию.

Значит, в клике $K$ есть еще одна вершина $w$, отличная от $x, y, z$. Как показано выше, $N^{*}(w)=K \bigcup\left\{w_{1}, w_{2}\right\}$, где $w_{1}, w_{2}$ - смежные вершины, не лежащие в клике $K$. По утверждению 3 в $N^{*}(w) \bigcap N^{*}(x)$ есть хотя бы одна вершина, не лежащая в $K$. Иначе говоря, $\{a, b, c\} \bigcap\left\{w_{1}, w_{2}\right\} \neq \varnothing$. Аналогично, $\{a, d\} \bigcap\left\{w_{1}, w_{2}\right\} \neq \varnothing$ и $\{b, d\} \bigcap\left\{w_{1}, w_{2}\right\} \neq \varnothing$. Легко видеть, что есть только два варианта с точностью до перемены местами $w_{1}, w_{2}: w_{1}$ совпадает с $d$ и $w_{2} \in\{a, b, c\}$ или $w_{1}$ совпадает с $a$ и $w_{2}$ совпадает с $b$.

Отметим, что если в клике $K$ есть еще одна вершина $w^{\prime}$, отличная от $x, y, z, w$, то аналогичными рассуждениями получаем, что $w^{\prime}$ смежна с двумя вершинами, лежащими вне клики $K$ : либо с вершинами $\{a, b\}$ (и в этом случае $N^{*}(w) \bigcap N^{*}\left(w^{\prime}\right)=K$, что противоречит утверждению 3 ), либо с вершинами $\{d, c\}$ (и тогда вершины $w$ и $w^{\prime}$ эквивалентны, что противоречит условию). Следовательно, в клике $K$ ровно 4 вершины, $\rho(G)=4, \Delta(G)=\rho(G)+2=6$.

Рассмотрим первый вариант, то есть $w_{1}$ совпадает с $d$ и $w_{2} \in\{a, b, c\}$. Если $w_{2}$ совпадает с $b$, то $N^{*}(w)=N^{*}(z)$, то есть вершины $w_{2}$ и $z$ эквивалентны, что невозможно. Аналогично $w_{2}$ не может совпадать с вершиной $a$. Следовательно, $w_{2}$ совпадает с вершиной $c$. Значит, вершины $c$ и $d$ смежны (рис. 3в). Но вершина $d$ смежна как минимум с 6 вершинами $(a, b, c, y, z, w)$, следовательно, $\operatorname{deg} d=6$. Но по условию есть только одна вершина максимальной степени - вершина $x$. Получаем противоречие.

Рассмотрим второй вариант, то есть $w_{1}$ совпадает с $a$ и $w_{2}$ совпадает с $b$. Тогда вершины $a$ и $b$ смежны. Рассмотрим ребро $(x, c)$. Если вершина $c$ не смежна ни c $b$, ни с $a$, то все вершины, соединенные ребрами с концами этого ребра, лежат в клике $K$, и по утверждению 2 ребро является собственным. Значит, существует ребро $(a, c)$ или $(b, c)$. Пусть, без ограничения общности, существует ребро $(b, c)$ (рис. 3г). Но тогда $\operatorname{deg} b=6$ (вершина $b$ смежна с вершинами $a, c, d, x, y, w)$, что снова приводит к противоречию.

Утверждение 11. Если $\rho(G)=\Delta(G)-2$ и в графе $G \in \Im$ существует ровно одна вершина максимальной степени, то граф $G$ получается из графа $B$ добавлением одной доминирующей вершины.

Доказательство. Снова используем обозначения, введенные в доказательстве утверждения 10: $x$ - единственная вершина максимальной степени; $K$ - клика наи- 
большего размера; по утверждению 9 вершина $x$ лежит в клике $K$; вершина $x$ смежна ровно с тремя вершинами $a, b, c$ графа $G$, не лежащими в клике $K$.

По утверждению $5 \rho(G) \geqslant 3$. Рассмотрим вершину $y$ клики $K$, отличную от $x$. По утверждению 10 вершина $y$ смежна с двумя вершинами из множества $\{a, b, c\}$. Пусть без ограничения общности это вершины $a$ и $b$. По утверждению 4 эти вершины смежны; тогда получаем, что множество вершин $\{a, b, x, y\}$ порождает клику порядка 4 в графе $G$. Следовательно, $\rho(G) \geqslant 4$.

В клике $K$ каждая вершина, отличная от $x$, соединена ребрами с парой вершин из множества $\{a, b, c\}$, причем в силу отсутствия эквивалентных вершин разные вершины соединены с разными парами. Следовательно, в клике $K$ может быть не более трех вершин, отличных от $x$. Учитывая, что $\rho(G) \geqslant 4$, получаем $\rho(G)=4$. $\mathrm{C}$ точностью до обозначений вершин граф $G$ выглядит следующим образом. Клика $K$ порождается множеством вершин $\{x, y, z, w\}$; вершина $x$ соединена ребрами с вершинами $a, b, c$; вершина $y-$ с вершинами $a, b$ (соответственно, вершины $a$ и $b$ смежны); вершина $z-$ с вершинами $b, c$ (вершины $b$ и $c$ смежны); вершина $w$ - с вершинами $a, c$ (вершины $a$ и $c$ смежны). Из всех возможных ребер в графе $G$ отсутствуют только ребра $(y, c),(z, a),(w, b)$ (рис. 4). Вершина $x$ является доминирующей в графе $G$. Удалим из графа $G$ вершину $x$ и все 6 инцидентных ей ребер Поскольку, как уже указано в начале статьи, граф $b$ получается при удалении из полного графа на 6 вершинах любых трех попарно не смежных ребер, понятно, что при удалении получается граф $b$. Легко убедиться в том, что граф $G$ сжат и не имеет собственных ребер.

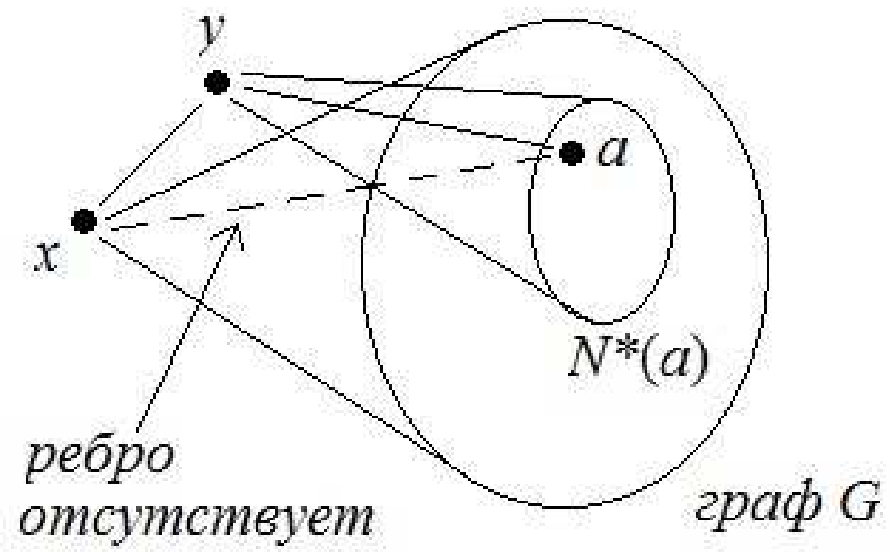

Рис. 4.

Утверждение 12. В классе $\Im$ с точностъю до изоморфизма существует единственный граф порядка не более 6: это граф $B$.

Доказательство. Если порядок графа $G \in \Im$ не превосходит 6 , то $\Delta(G) \leqslant 5$. Если $\Delta(G) \leqslant 4$, то согласно утверждению 8 граф $G$ изоморфен графу $b$. Остается рассмотреть случай $\Delta(G)=5$. При таком условии порядок графа равен 6 и в графе $G$ существует вершина максимальной степени $x$, причем в данном случае это будет доминирующая вершина $(\operatorname{deg} x=\Delta(G)=5)$. Очевидно, что две различные доминирующие 
вершины в графе эквивалентны друг другу, поэтому $x$ - единственная вершина графа $G$ максимальной степени. По утверждению 5 имеем $3 \leqslant \rho(G) \leqslant \Delta(G)-1=4$. Если $\rho(G)=3=\Delta(G)-2$, то попадаем в условие утверждения 11 и получаем, что граф $G$ получается из графа $b$ добавлением доминирующей вершины и поэтому должен иметь 7 вершин, что приводит к противоречию. Следовательно, $\rho(G)=4=\Delta(G)-1$. Но тогда $\Delta(G) \leqslant 4$ по утверждению 7 , что снова приводит к противоречию.

Утверждения 7, 8 и 12 доказывают теорему 1, сформулированную во введении. Утверждение 11 есть теорема 2.

\section{Операция расширения графа}

Рассмотрим следующую операцию над произвольным графом $G$, которую назовем операцией расширения с центром в вершине $a$. Пусть $a$ - произвольная вершина этого графа. Добавим к графу $G$ две новые вершины $x$ и $y$, соединив ребрами вершину $x$ со всеми вершинами графа $G$, за исключением вершины $a$, и вершину $y$ со всеми вершинами из замкнутого окружения $N^{*}(a)$. Иллюстрация операции расширения приведена на рис. 5 .

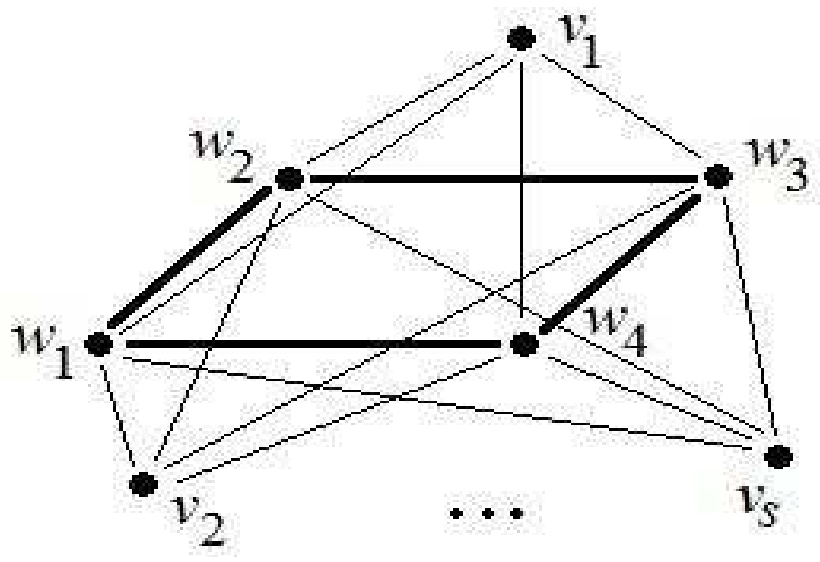

Рис. 5.

Рассмотрим свойства операции расширения. Начнем с элементарного описания окружений всех вершин.

Утверждение 13. Пусть граф $F$ получен из графа $G$ с множеством вершин $V$ однократным применением операчии расширения с иентром в вершине $a \in V$. Тогда $N_{F}^{*}(a)=N_{G}^{*}(a) \bigcup\{y\}, N_{F}^{*}(x)=(V \backslash\{a\}) \bigcup\{x, y\}, N_{F}^{*}(y)=N_{G}^{*}(a) \bigcup\{x, y\}, N_{F}^{*}(z)=$ $N_{G}^{*}(z) \bigcup\{x, y\}$, если $z \in N_{G}(a), N_{F}^{*}(z)=N_{G}^{*}(z) \bigcup\{x\}$, если $z \in V \backslash N_{G}(a)$.

Доказательство следует из описания операции расширения.

Теперь изучим возможности наличия в построенном графе эквивалентных пар вершин.

Утверждение 14. Пусть граф F получен из графа $G \in \Im$ однократным применением операции расширения с иентром в вершине а. Тогда граф $F$ является сжатым. 
Доказательство. Сначала докажем, что вершина $x$ не эквивалентна никакой другой вершине графа $F$. Предположим, что существует вершина $w$ графа $F$, эквивалентная вершине $x$. Тогда вершина $w$ не совпадает с $a$ (поскольку вершины $x$ и $a$ не смежные) и вершина $w$ не совпадает с $y$ (поскольку $y$ смежна с $a$, а $x$ нет). Так как $y \in N_{F}^{*}(x)$ и, по определению эквивалентности, $N_{F}^{*}(x)=N_{F}^{*}(w)$, то $y \in N_{F}^{*}(w)$. Но тогда вершина $y$ смежна с вершиной $w$, то есть $w \in N_{F}^{*}(y)$. Далее, $N_{F}^{*}(y)=N_{G}^{*}(a) \bigcup\{x, y\}$ в силу утверждения 13 и $w$ отлична от вершин $a, x, y$, поэтому $w \in N_{G}(a)$. Но тогда вершины $w$ и $a$ смежны и, следовательно, $a \in N_{F}(w)$. Значит, $a \in N_{F}^{*}(w)$, но по определению операции расширения $a \notin N_{F}^{*}(x)$. То есть равенство $N_{F}^{*}(x)=N_{F}^{*}(w)$ не может быть выполнено, что приводит к противоречию.

Теперь покажем, что вершина $y$ не эквивалентна никакой другой вершине графа $F$. Поскольку вершина $x$ уже рассмотрена, надо доказать, что $y$ не эквивалентна какой-либо вершине $v$ графа $G$. Действительно, в противном случае, вершина $v$ отлична от $a$, так как вершина $x$ смежна с $y$ и, в силу эквивалентности вершин $y$ и $v$, должна быть смежна с $v$, а вершины $a$ и $x$ не смежные. Далее, $N_{F}^{*}(v)=N_{F}^{*}(y)=$ $N_{G}^{*}(a) \bigcup\{x, y\}$ по определению эквивалентности и утверждению 13, и имеем цепочку равенств множеств $N_{G}^{*}(v)=N_{F}^{*}(v) \backslash\{x, y\}=N_{F}^{*}(y) \backslash\{x, y\}=N_{G}^{*}(a)$. Следовательно, вершины $v$ и $а$ различны и эквивалентны в графе $G$, что противоречит условию $G \in \Im$.

Вершина $a$ не может быть эквивалентна в графе $F$ какой-либо другой вершине графа $G$, поскольку все такие вершины смежны с $x$, а вершина $a$ нет.

Наконец, пусть $v$ и $w$ - две различные вершины графа $G$, отличные от вершины $a$ и эквивалентные в графе $F$. Отметим, что $x \in N_{F}^{*}(v)=N_{F}^{*}(w)$. Далее, вершина $a$ либо смежна с обеими вершинами $v$ и $w$, либо не смежна также с обеими. В первом случае вершина $y$ по построению также оказывается смежной вершинам $v$ и $w$, и тогда $N_{G}^{*}(v)=N_{F}^{*}(v) \backslash\{x, y\}=N_{F}^{*}(w) \backslash\{x, y\}=N_{G}^{*}(w)$; во втором случае вершина $y$ не смежная с обеими вершинами $v$ и $w$, и $N_{G}^{*}(v)=N_{F}^{*}(v) \backslash\{x\}=N_{F}^{*}(w) \backslash\{x\}=$ $N_{G}^{*}(w)$. В обоих случаях получаем, что в графе $G$ вершины $v$ и $w$ эквивалентны, что невозможно.

Теперь рассмотрим граф, полученный при операции расширения, с точки зрения существования в нем свободных ребер. Сначала докажем пару простых утверждений.

Утверждение 15. Пусть граф $F$ получен из графа $G$ операцией расширения с иентром в вершине а. Если $K$ - максимальная клика графа $G$, то либо вершина а лежит в $K$ (и тогда $K \bigcup\{y\}$ является максимальной кликой графа $F$ ), либо вершина а не лежит в $K$ (и тогда $K \bigcup\{x\}$ является максимальной кликой графа $F)$.

Доказательство. Пусть $K$ - максимальная клика графа $G$. Тогда в графе $F$, очевидно, $K$ останется кликой (но не обязательно максимальной), поскольку при операции расширения ребра из графа не удалялись. Кроме того, так как при операции расширения не добавлялись ребра, соединяющие вершины графа $G$, то к клике $K$ не добавится никакая другая вершина графа $G$. То есть вершинами, которые могут добавиться к клике $K$ в графе $F$, являются новые вершины $x$ и $y$. Если вершина $a$ лежит в $K$, то все вершины клики $K$ смежны с $a$, то есть $K \subset N_{G}^{*}(a)$. Следовательно, в графе $F$ со всеми вершинами клики $K$ смежна вершина $y$, а вершина $x$ не смежна с вершиной $a$. Значит, $K \bigcup\{y\}$ является кликой в графе $F$, а поскольку к ней нельзя добавить ни вершину $x$, ни какую-нибудь другую вершину графа $G$, то эта клика максимальна. Если же вершина $a$ не принадлежит клике $K$, то $K \not \subset N_{G}(a)$, 
поскольку в противном случае $K \bigcup\{a\}$ было бы кликой графа $G$, что противоречит максимальности $K$. Следовательно, в графе $F$ со всеми вершинами клики $K$ смежна вершина $x$, но не вершина $y$. То есть $K \bigcup\{x\}$ - клика графа $F$, и поскольку к ней нельзя добавить новую вершину графа $G$, то эта клика максимальна.

Утверждение 16. Если граф $G$ связен и не содержит собственных ребер, то всякая вершина графа $G$ принадлежит как минимум двум различным максимальным кликам графа $G$.

Доказательство. Если вершина а содержится только в максимальной клике $K$ графа $G$, то всякое ребро $(a, b)$, где $b$ - произвольная вершина клики $K$, отличная от $a$, лежит только в клике $K$, то есть является собственным ребром графа $G$. Отметим, что в силу связности графа $G$ хотя бы одно ребро такого вида существует.

Теперь докажем отсутствие собственных ребер в графе, полученном операцией расширения.

Утверждение 17. Пусть граф $F$ получен из графа $G \in \Im$ однократным применением операчии расширения с иентром в вершине а. Тогда граф $F$ не содержит собственных ребер.

Доказательство. Пусть $(v, w)$ - произвольное ребро графа $G$. Тогда, поскольку $G \in \Im$, существуют две различные максимальные клики графа $G$, содержащие ребро $(v, w)$. По утверждению 15 каждая из этих клик дополнится до максимальной клики графа $F$ либо вершиной $x$, либо вершиной $y$. В любом случае эти две новые максимальные клики будут, очевидно, различны, и ребро $(v, w)$ принадлежит обеим кликам. По определению ребро $(v, w)$ не является собственным ребром графа $F$.

Остается доказать, что не являются собственными следующие ребра: ребро $(x, y)$; ребра вида $(x, w)$, где вершина $w$ лежит в графе $G$ и отлична от вершины $a$; ребро $(y, a)$; ребра вида $(y, v)$, где вершина $v$ лежит в $N_{G}(a)$.

Окружение $N_{G}(a)$ вершины $a$ в графе $G$ не является полным подграфом, поскольку в противном случае множество вершин $N_{G}^{*}(a)$ порождает в графе $G$ клику $K$. Эта клика в графе $G$ обязана быть максимальной, так как вершина $a$ принадлежит клике $K$ и все вершины, смежные с вершиной $a$, лежат в $N_{G}(a)$. Но всякая клика (не обязательно максимальная) графа $G$, содержащая вершину $a$, по этой же причине содержится в $N_{G}^{*}(a)$, а значит, в клике $K$, и поэтому клика $K$ - единственная максимальная клика графа $G$, содержащая вершину $a$.

Рассмотрим ребро $(x, y)$. Согласно доказанному выше в окружении $N_{G}(a)$ вершины $a$ найдется пара не смежных вершин. По определению операции расширения каждая из этих двух вершин смежна и с $x$, и с $y$. По утверждению 2 ребро $(x, y)$ не является собственным ребром графа $F$.

Далее рассмотрим любое ребро $(x, w)$, где вершина $w$ лежит в графе $G$ и отлична от вершины $a$. Пусть сначала вершина $w$ не лежит в $N_{G}(a)$. Граф $G \in \Im$, следовательно, в силу связности найдется вершина $о$, смежная с $w$, и в силу отсутствия собственных ребер в графе $G$ ребро $(w, o)$ лежит хотя бы в двух максимальных кликах. Поэтому и вершина $w$ содержится как минимум в двух различных максимальных кликах $K$ и $L$ графа $G$. Эти клики не содержат вершину $a$, так как вершина $w$ не смежна с ней, и по утверждению 15 клики $K \bigcup\{x\}, L \bigcup\{x\}$ максимальны в графе $F$. Так как обе указанные клики различны и содержат ребро $(x, w)$, то указанное ребро не является собственным ребром графа $F$. 
Пусть теперь вершины $w$ и $a$ смежные, то есть $w \in N_{G}(a)$. Ребро $(a, w)$ не является собственным ребром графа $G$, поэтому существуют две различные максимальные клики $K$ и $L$ графа $G$, содержащие это ребро. Так как эти клики различны, то в графе $G$ существуют такие несмежные вершины $k$ и $l$, что $k$ лежит в $K, l$ лежит в $L$. Поскольку вершина $a$ отлична от этих двух вершин, в графе $F$ вершины $K$ и $l$ соединены ребрами с вершиной $x$. Значит, каждая из вершин $K$ и $l$ смежна с обоими концами ребра $(x, w)$ и при этом они не соединены между собой. По утверждению 2 ребро $(x, w)$ не является собственным ребром графа $F$.

Наконец рассмотрим ребро $(y, v)$, где вершина $v$ лежит в $N_{G}(a)$. В графе $G$ ребро $(a, v)$ не собственное, следовательно, существуют две различные максимальные клики графа $G$, содержащие это ребро. По утверждению 15 каждая из указанных клик дополнится в графе $F$ до максимальной клики добавлением вершины $y$. Поскольку при добавлении получатся, очевидно, различные клики графа $F$, имеем две различные максимальные клики графа $F$, содержащие ребро $(y, v)$. Кроме того, каждая из этих двух клик графа $F$ содержит ребро $(y, a)$, так как содержит обе вершины $y$ и $a$. Значит, ребро $(y, a)$ также не собственное.

Объединяя утверждения 14 и 17 и замечая, что при применении операции расширения к связному графу получится, очевидно, связный граф, получаем следующее.

Утверждение 18. Пусть граф $F$ получен из графа $G \in \Im$ однократнъм применением операчии расширения с иентром в вершине а. Тогда граф $F \in \Im$.

Укажем далее, как изменяются такие параметры графа, как количество вершин, плотность и максимальная степень при применении операции расширения.

Утверждение 19. Пусть граф $F$ получен из графа $G \in \Im$ порядка $n$ однократным применением операции расширения с иентром в вершине а. Тогда $\rho(F)=\rho(G)+1$ $u \Delta(F)=n$.

Доказательство. Граф $F$ имеет $n+2$ вершины. Вершина $x$ смежна в графе $F$ со всеми остальными вершинами, за исключением $a$. Поэтому $\Delta(F)=n$. Из утверждения 15 получаем, что в графе $F$ есть клика порядка $\rho(G)+1$. Поскольку ребер, соединяющих вершины графа $G$, при операции расширения не добавлялось, клики графа $F$, состоящие только из вершин графа $G$, могут иметь не более $\rho(G)$ вершин. Но так как новых вершин в $F$ всего две (это $x$ и $y$ ), то $\rho(F) \leqslant \rho(G)+2$. Предположим, что $\rho(F)=\rho(G)+2$ и $K$ - наибольшая клика графа $F$. В силу сказанного выше $\{x, y\} \subset K$, и тогда $L=K \backslash\{x, y\}$ порождает клику порядка $\rho(G)$ в графе $G$. Но из утверждения 13 легко следует, что $l$ содержится в окружении $N_{G}(a)$. Значит, вершина $a$ смежна со всеми вершинами клики $l$ и $L \bigcup\{a\}$ порождает клику порядка $\rho(G)+1$ в графе $G$, что невозможно.

Что будет при многократном применении операции расширения? Если начать с графа $G_{0} \in \Im$ с $n_{0}$ вершинами и плотностью $\rho_{0}$, то результатом применения операции расширения к графу $G_{0}$ (центр расширения - вершину $a$ выбираем произвольно) будет граф $G_{1} \in \Im$ с $n_{0}+2$ вершинами, плотностью $\rho_{0}+1$ и максимальной степенью $n_{0}$. Применяя операцию к графу $G_{1}$, получаем граф $G_{2} \in \Im$ с $n_{0}+4$ вершинами, плотностью $\rho_{0}+2$ и максимальной степенью $n_{0}+2$. Продолжая далее, индуктивно строим граф $G_{t} \in \Im, t \geqslant 1$, с $n_{0}+2 t$ вершинами, плотностью $\rho_{0}+t$ и максимальной степенью $n_{0}+2(t-1)$. Таким образом, доказано следующее. 
Утверждение 20. Пусть существует граф $G_{0} \in \Im$ с $n_{0}$ вершинами и плотностью $\rho_{0}$. Тогда для любого $t \geqslant 1$ в классе $\Im$ существует граф с $n_{0}+2 t$ вершинами, плотностъю $\rho_{0}+t$ и максималъной степенъю $n_{0}+2(t-1)$.

Теперь для произвольного натурального значения $s \geqslant 2$ рассмотрим граф $B_{s}$ с множеством вершин $\left\{v_{1}, \ldots, v_{s}, w_{1}, w_{2}, w_{3}, w_{4}\right\}$ и множеством ребер $\left\{\left(v_{i}, w_{j}\right): i=\right.$ $\overline{1, s}, j=1,2,3,4\} \bigcup\left\{\left(w_{1}, w_{2}\right),\left(w_{2}, w_{3}\right),\left(w_{3}, w_{4}\right),\left(w_{4}, w_{1}\right)\right\}$ (см. рис. 6$)$.

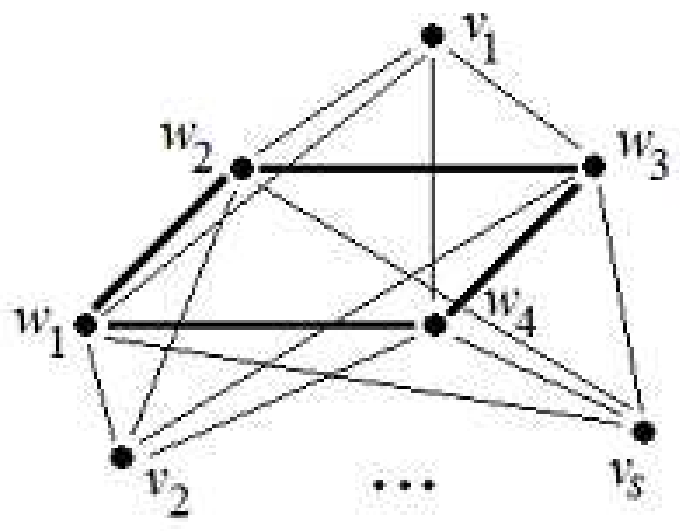

Рис. 6.

Граф $B_{s}$, очевидно, связен. Пар эквивалентных вершин в нем нет, поскольку $N^{*}\left(v_{i}\right)=\left\{v_{i}, w_{1}, w_{2}, w_{3}, w_{4}\right\}$ для любого $i=\overline{1, s}, N^{*}\left(w_{1}\right)=\left\{w_{1}, w_{2}, w_{4}\right\}$, $N^{*}\left(w_{2}\right)=\left\{w_{1}, w_{2}, w_{3}\right\}, N^{*}\left(w_{3}\right)=\left\{w_{2}, w_{3}, w_{4}\right\}, N^{*}\left(w_{4}\right)=\left\{w_{1}, w_{3}, w_{4}\right\}$. Ребро $\left(w_{1}, w_{2}\right)$ лежит как минимум в двух максимальных кликах, порожденных множествами вершин $\left\{w_{1}, w_{2}, v_{1}\right\},\left\{w_{1}, w_{2}, v_{2}\right\}$. Ребро $\left(v_{1}, w_{1}\right)$ лежит в двух максимальных кликах $\left\{w_{1}, w_{2}, v_{1}\right\},\left\{w_{1}, w_{4}, v_{1}\right\}$. Остальные ребра графа $B_{s}$ по аналогичным причинам не являются собственными. Значит, $B_{s} \in \Im$.

Число вершин в графе $B_{s}$ равно $s+4$, его плотность равна 3 , максимальная степень равна $s+2$. Отметим, что граф $B_{2}$ изоморфен графу $b$, приведенному на рис.1. Поскольку $s \geqslant 2$ - любое, в классе $\Im$ существует граф плотности 3 и произвольной максимальной степени $\Delta \geqslant 4$.

Если взять в качестве исходного графа $G_{0}$ граф $B_{s}$, то получим после $t$ итераций граф с $2 t+s+4$ вершинами, плотностью $t+3$ и максимальной степенью $2 t+s+2$. То есть для любого значения $\rho=t+3>3$ в классе $\Im$ существует граф с плотностью $\rho$ и максимальной степенью $2 \rho+s-4$. Учитывая, что $s \geqslant 2$ можно выбирать любым натуральным числом, большим 1 , получаем, что в классе $\Im$ существует граф с с плотностью $\rho$ и максимальной степенью $\Delta \geqslant 2 \rho-2$. Таким образом, получаем теорему 3, сформулированную во введении.

Сделаем следующее замечание. Над графами из класса э существует довольно легко обнаруживаемая операция: взять два непересекающихся графа из э и отождествить в них два изоморфных порожденных подграфа. В самом простом виде отождествить любую вершину одного графа с произвольной вершиной другого графа. Такая операция не увеличивает плотность графа по сравнению с плотностью исходных графов, но увеличивает максимальную степень. Поэтому в настоящей работе и предложена операция расширения графа, носящая не самый очевидный 
характер, но увеличивающая плотность графа по сравнению с исходным. Оценка $\Delta \geqslant 2 \rho-2$ в теореме 3 может быть улучшена, если удастся построить такую операцию над графами из класса $\Im$, которая увеличивает плотность графа на число $d$ и максимальную степень графа на число, меньшее $2 d$.

\section{Список литературы}

1. Ролдугин П. В., Редукция графа при поиске реберного покрытия минимальным числом клик. Математические вопросъ криптографии (2012) 3, №3, 105-128.

2. Cavers M. S., Clique partitions and coverings of graphs, An essay presented to the University of Waterloo in fulfillment of the essay requirement for the degree of Masters of Mathematics. Waterloo, Ontario, Canada, December 20th, 2005..

3. Gramm J., Guo J., Huffner F., Niedermeier R., Data Reduction, Exact, and Heuristic Algorithms for Clique Cover. Proc. 8th ALENEX-06 (2006) 86-94.

4. Kou L. T., Stockmeyer L. J., Wong C. K., Cliques with Regard to Keyword Conflicts and Intersection Graphs. Comm. ACM (Feb. 1978) 21, №2,.

5. Orlin J., Contentment in graph theory: Covering graphs with cliques. Indagat. Math. (1977) 39, 406-424.

Статья поступила 16.05.2013. 